\section{The species-specific egg receptor for sea urchin sperm adhesion is EBR1, a novel ADAMTS protein}

\author{
Noriko Kamei ${ }^{1}$ and Charles G. Glabe \\ Department of Molecular Biology and Biochemistry, \\ University of California, Irvine, Irvine, California 92697, USA
}

Species-specific adhesion of sperm to the egg during sea urchin fertilization involves the interaction of the sperm adhesive protein, bindin, and a complementary receptor on the egg surface, and serves to restrict the gene pool to individuals of the same species. We used PCR representation difference analysis to clone the species-specific egg receptor for bindin, EBR1, from Strongylocentrotus franciscanus $(S f)$ and $S$. purpuratus $(S p)$. Sf-EBR1 contains a novel ADAMTS-like $\mathrm{N}$-terminal domain followed by $\sim 19$ tandem EBR repeats consisting of alternating CUB and thrombospondin type 1 (TSP-1) domains where the last $10 \mathrm{EBR}$ repeats are species-specific and highly conserved. Recombinant protein corresponding to the species-specific EBR repeat displays species-specific sperm adhesion and bindin-binding activity. The $S p$ EBR1 ortholog has the same ADAMTS (a disintegrin and metalloprotease with thrombospondin type-1 modules) core region followed by eight and one-half tandem egg bindin receptor (EBR) repeats that share $88 \%$ identity with the $S f$-EBR1 repeats, but has an entirely different species-specific domain consisting of hyalin-like (HYR) repeats. Thus, the species-specific domains of egg bindin receptor 1 (EBR1) from both species function as the egg surface receptor to mediate species-specific sperm adhesion.

Received July 14, 2003; revised version accepted August 25, 2003.

Sperm-egg interaction typically exhibits species specificity in most animals. Several molecules from both sperm and eggs that are thought to mediate gamete interaction have been identified in many animals. In abalone, the acrosomal protein of sperm, lysin, interacts with the egg vitelline envelope receptor for lysin (VERL) and creates a hole on the extracellular matrix (ECM) of the egg with species selectivity (Swanson and Vacquier 1997). In mammals, numerous molecules, such as galactosyltransferase, ADAM (a disintegrin and metalloprotease), and Sp56, have been identified from sperm and are proposed to interact with such as egg zona pellucida pro-

[Keywords: EBR1; ADAMTS; TSP-1; Hyalin; sea urchin; species specificity]

${ }^{1}$ Corresponding author.

E-MAIL nkamei@uci.edu; FAX (949) 824-8551.

Article and publication are at http://www.genesdev.org/cgi/doi/10.1101/ gad.1133003. teins Zp2, Zp3, or Integrins on the egg. However, a clear understanding of the specificity of these interactions and the regulation of species specificity is not yet known (Primakoff and Myles 2002; He et al. 2003).

Acrosome-reacted sea urchin sperm adhere to glycoprotein receptors that are components of the vitelline envelope (VE) known as the ECM of the egg. It has been known for several decades that the protein bindin on the acrosome-reacted sea urchin sperm head mediates the species-specific adhesion of sperm to the VE during fertilization (Summers and Hylander 1975; Glabe and Vacquier 1977b; Vacquier and Moy 1977; Glabe et al. 1982). The egg ECM also serves as a major barrier for interspecific fertilization in mammalian systems (Yanagimachi $1981,1994)$. In sea urchins, the complementary receptor for bindin was shown to be an $\sim 350-\mathrm{kD}$ protein on the VE of the egg (Glabe and Vacquier 1978; Carroll et al. 1986; Ruiz-Bravo and Lennarz 1986; Ohlendieck et al. 1993). However, the identity of this receptor has remained elusive, and attempts to solubilize and purify the active receptor from the VE of the egg have not been successful (Glabe and Lennarz 1981; Ruiz-Bravo et al. 1986). Therefore, we used a novel molecular approach, representation difference analysis (RDA), to amplify species-specific cDNA fragments from ovaries of the sea urchin Strongylocentrotus franciscanus $(S f)$ that are distinct from cDNAs fragments in another species of the same genus, $S$. purpuratus $(S p)$, to identify genes involved in determining species specificity (Hubank and Schatz 1994; Kamei et al. 2000).

Here we report the identification and characterization of the species-specific egg bindin receptor (EBR1) from two sea urchin species, $S$. franciscanus and $S$. purpuratus, along with their unique domain structures. The biological activity of recombinant species-specific egg bindin receptor (EBR) and hyalin-like (HYR) repeats indicate that they serve as species-specific ligands for the sperm adhesive protein bindin.

\section{Results and Discussion}

Characterization of the species-specific cDNA clone, Sf-H2

We isolated four species-specific cDNA clones from $S$. franciscanus ovary mRNA, as previously described (Kamei et al. 2000). One of the clones (Sf-H2) was a candidate for the egg-bindin receptor because it hybridizes species-specifically to an $\sim 14-15-\mathrm{kb}$ mRNA (see below) and therefore is sufficiently large to encode the $350-\mathrm{kD}$ egg receptor protein. We raised polyclonal antibodies against recombinant $S f-\mathrm{H} 2$ protein, and the antibodies detect a protein of $\sim 350 \mathrm{kD}$ on purified VEs from $S$. franciscanus, but not $S$. purpuratus eggs (Fig. 1A,B). Thus, the $S f-\mathrm{H} 2$ cDNA probe and antibody detect molecules of the size predicted for the egg receptor.

Because the $S f-\mathrm{H} 2$ transcript encodes a species-specific VE protein of the size and location expected for the receptor, we determined whether $S f-\mathrm{H} 2$ displayed speciesspecific adhesive activity by using several different assays. We first examined the effect of anti-Sf-H2 antibody or recombinant $S f-\mathrm{H} 2$ protein on fertilization. Anti- $S f$ $\mathrm{H} 2$ polyclonal antibody inhibited fertilization of $S$. franciscanus eggs in a concentration-dependent manner, but 
A

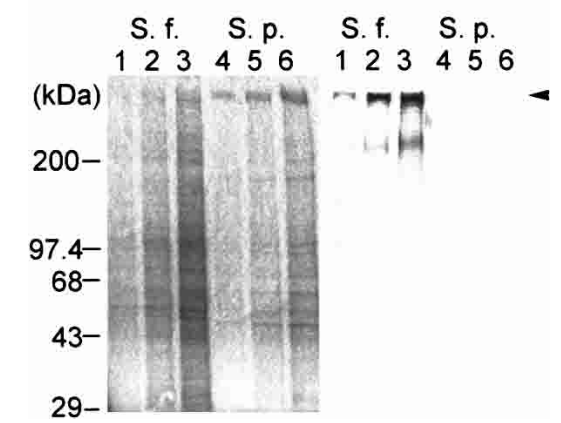

Figure 1. Species specificity of $S f-\mathrm{H} 2 .(A)$ Coomassie blue staining of purified VEs from $S$. franciscanus and $S$. purpuratus separated on a $4 \%-20 \%$ SDS-PAGE gel. (Lanes 1-3) S. franciscanus VE. (Lanes 4-6) S. purpuratus VE. (B) Western blot analysis of VE from $S$. franciscanus and $S$. purpuratus separated on a $4 \%-20 \%$ SDS-PAGE gel (shown in A) using anti-Sf-H2 antibody. The arrow highlights the $\sim 350-\mathrm{kD}$ protein detected by the anti-Sf-H2 antibody. (Lanes 1-3) $S$. franciscanus VE. (Lanes 4-6) S. purpuratus VE. Lanes 1 and 4 are $2 \mu \mathrm{g}$ of VE protein, lanes 2 and 5 are $5 \mu \mathrm{g}$ of VE protein, and lanes 3 and 6 are $10 \mu \mathrm{g}$ of $\mathrm{VE}$ protein.

had no effect on the fertilization of $S$. purpuratus eggs (Fig. 2A). Similarly, recombinant $S f-\mathrm{H} 2$ protein only inhibited S. franciscanus fertilization, but not S. purpuratus fertilization (Fig. 2B). These results suggest that anti$S f-\mathrm{H} 2$ antibodies block the receptor on the egg, and the recombinant $S f-\mathrm{H} 2$ protein competitively inhibits the binding of sperm to the VE receptor. We also directly examined sperm adhesive activity of $S f-\mathrm{H} 2$ by using a recombinant glutathione $S$ transferase (GST) fusion protein immobilized on glutathione agarose beads to assess sperm adhesion to the beads. We found that acrosomereacted $S$. franciscanus sperm specifically bound to beads containing immobilized $S f$-H2 GST fusion protein, but not to control beads containing GST alone (Fig. 2C). These data are consistent with the interpretation that $S f-\mathrm{H} 2$ encodes a species-specific sperm receptor.

The sperm adhesive protein bindin is commonly purified as insoluble acrosomal granules, and these bindin granules were shown to species-specifically agglutinate eggs of the same species (Glabe and Lennarz 1979). This agglutination activity of bindin granules can be used to measure the adhesive interaction between bindin and the egg surface (Glabe and Lennarz 1979). Recombinant $S f-\mathrm{H} 2$ protein specifically inhibits agglutination of $S$. franciscanus eggs by homologous bindin, but does not inhibit the agglutination of $S$. purpuratus eggs by $S$. purpuratus bindin (Fig. 2D). We also directly measured the interaction of iodinated recombinant $S f-\mathrm{H} 2$ protein to purified bindin granules using a sedimentation assay. Recombinant $S f-\mathrm{H} 2$ protein bound only to $S$. franciscanus bindin granules in a concentration-dependent and saturable manner (Fig. 2E). Therefore, $S f$-H2 satisfies a number of different functional criteria for the egg surface adhesive receptor for bindin.

The egg surface receptor for sperm is either released from the VE or destroyed due to the action of proteases that are released from the cortical granules upon fertilization (Vacquier et al. 1973; Glabe and Vacquier 1977a). We examined the localization of the Sf-H2 on whole mounts of fertilized and unfertilized eggs by immunofluorescence staining with anti-Sf-H2 antibodies. Bright staining was detected on the unfertilized egg surface, but
A

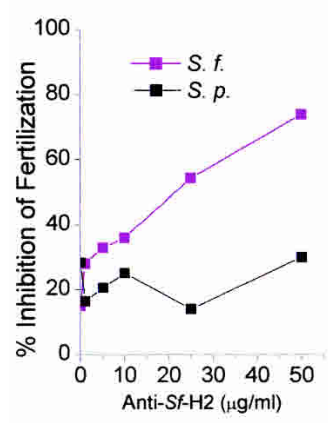

C

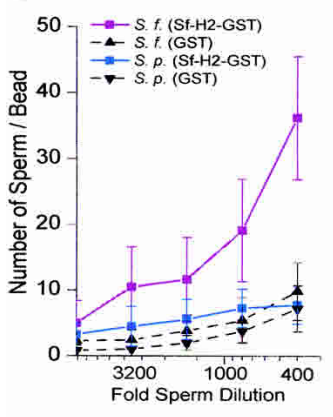

E

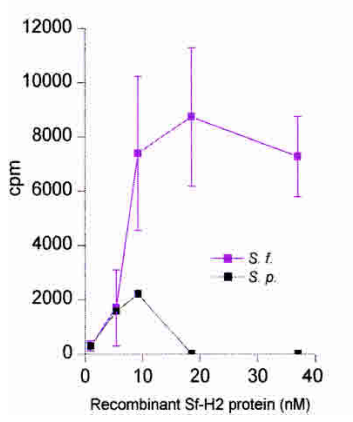

B

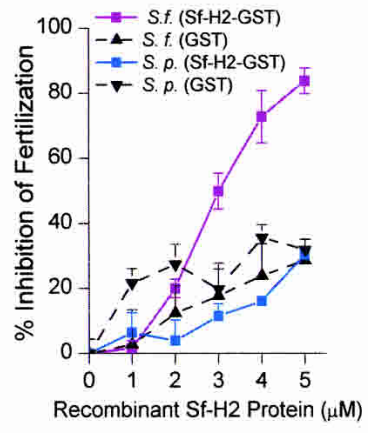

D

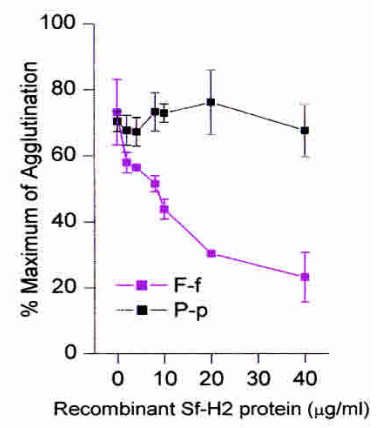

$\mathbf{F}$

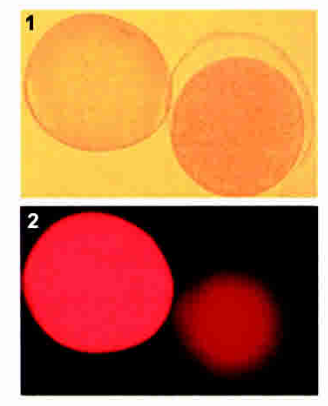

Figure 2. Inhibition of fertilization by anti-Sf-H2 antibody and recombinant $S f-\mathrm{H} 2$ protein. (A) Species-specific inhibition of S. franciscanus fertilization by anti-Sf-H2 antibody. Increasing concentrations of the antibody only inhibit the fertilization of homologous eggs. Red line indicates $S$. franciscanus gametes; black line indicates $S$. purpuratus gametes. $(B)$ Inhibition of fertilization by recombinant $S f-H 2$. Increasing concentrations of $S f-\mathrm{H} 2$ inhibits only the fertilization of homologous eggs. Both red and blue lines show the percentage of inhibition of fertilization by using recombinant $S f$-H2-GST. Both broken lines indicate control samples GST only. (C) Speciesspecific binding of acrosome-reacted sperm to immobilized recombinant $S f-\mathrm{H} 2$ protein. The graph shows the number of sperm binding to the beads. Red line indicates $S$. franciscanus sperm; blue line, $S$. purpuratus sperm. Both broken lines show the binding to GST controls. (D) Species-specific inhibition of bindin-mediated egg agglutination by recombinant $S f-\mathrm{H} 2$ protein. The percentage of agglutinated eggs is shown as a function of increasing recombinant $S f-H 2$ protein. Red line indicates $S$. franciscanus eggs and S. franciscanus bindin; black line indicates $S$. purpuratus eggs and $S$. purpuratus bindin. $(E)$ Species-specific binding of recombinant iodinated $S f-\mathrm{H} 2$ to purified bindin particles. Red line indicates $S$. franciscanus bindin; black line, $S$. purpuratus bindin. $(F)$ Immunostaining of unfertilized and fertilized $S$. franciscanus eggs with anti-Sf-H2 antibody. Panel 1 shows the phase contrast image; panel 2, the bright-field immunofluorescence staining with anti-Sf-H2 antibody. The egg on the left is unfertilized, and the one on the right is fertilized. 
this immunoreactivity was greatly diminished on the fertilization envelope and egg surface after fertilization (Fig. 2F). The absence of anti-Sf-H2 staining in the fertilization envelope of fertilized eggs is consistent with the known release or destruction of the receptor from the elevating fertilization envelope after fertilization.

The $S f-\mathrm{H} 2$ gene product meets all of the known structural and functional criteria for the egg receptor, including size, VE localization and modification after fertilization, species-specific sperm, and bindin-binding activity. Thus, from these data, we conclude that $S f-\mathrm{H} 2$ is the egg receptor for sperm, and we have named this gene product egg bindin receptor (EBR1).

Comparison of the sequences of EBR1 from both $\mathrm{S}$. franciscanus and $\mathrm{S}$. purpuratus

The deduced amino acid sequence of $S f$-EBR1 consists of 4595 amino acids (GenBank accession no. AY138966). Starting at the $\mathrm{N}$ terminus, the protein contains a 30 residue signal peptide followed by a putative propeptide, metalloprotease domain, cis-rich domain, and eight thrombospondin type 1 (TSP-1) domains, indicating that EBR1 shares several significant structural features with members of the ADAMTS family of proteins (a disintegrin and metalloprotease with thrombospondin type-1 modules; Tang and Hong 1999; Tang 2001). However, $S f$-EBR1 lacks a disintegrin-like domain that typically follows the catalytic domain in ADAMTS family members. $S f$-EBR 1 also contains an additional C-terminal region containing $\sim 19$ repeats of a novel motif (EBR repeats) located immediately after the ADAMTS-like core sequence (Fig. 3A). These novel motifs contain a TSP-1 and a CUB $(\mathrm{C} 1 \mathrm{~s} / \mathrm{C} 1 \mathrm{r}, \mathrm{uEGF}$ and bone morphogenic protein) subdomain (Bork and Beckmann 1993; Adams 1997). Each TSP-1 domain is from 53 to 59 amino acids, and each CUB domain is $\sim 104-123$ amino acid residues.
Thus, each EBR repeat is $\sim 513$ bp or 171 amino acids. The nine EBR repeats that follow immediately after the ADAMTS-like core region of Sf-EBR1 are significantly more variable than the last 10 EBR repeats and are defined as core EBR repeats because they also occur in $S p$ EBR1 (TSP, thin light blue ovals; CUB, yellow ovals; Fig. $3 \mathrm{~A})$. The last 10 EBR repeats of $S f$-EBR1 are homogeneous (TSP, thin green ovals; CUB, dark blue ovals), and each domain shows $85 \%-100 \%$ identity with the other repeats in this region. The last 10 homogeneous repeats are also species-specific, because they do not hybridize to $S$. purpuratus mRNA and are absent in $S p$-EBR1 (see below). The original cDNA fragment isolated by RDA, $S f$ $\mathrm{H} 2$, corresponds to one of these species-specific EBR repeats. In contrast, the TSP-1 domains outside of the EBR repeats in the ADAMTS-like core region and the TSP-1 subdomains present in the first nine core EBR repeats (thin light blue ovals) contain only $12 \%-50 \%$ identity with the TSP-1 subdomains in the species-specific EBR repeats (green ovals). Similarly, the first nine and the last CUB subdomains that are in the EBR repeat /yellow ovals) display only $11 \%-20 \%$ identity with the CUB subdomains in the species-specific EBR repeats (dark blue ovals; Fig. 3A). The second core EBR repeat for $S$. franciscanus (also for $S$. purpuratus; see Fig. 3A) has a duplicated CUB domain in contrast with all other EBR repeats, which have one CUB domain.

Although the species-specific $S f$-EBR1 repeats do not hybridize with $S$. purpuratus DNA, $S$. purpuratus has a homologous EBR1 gene because the 3' UTR of $S f$-EBR1 hybridizes to the same size fragment on genomic Southern blots of $S$. purpuratus DNA (data not shown). The EBR1 gene was cloned and sequenced from an $S$. purpuratus ovary cDNA library by using the $3^{\prime}$ UTR of $S f$ EBR 1 as an initial probe and the same cloning and sequencing strategy used for Sf-EBR1 (GenBank accession no. AY341264). The gene encodes a protein with a strik-

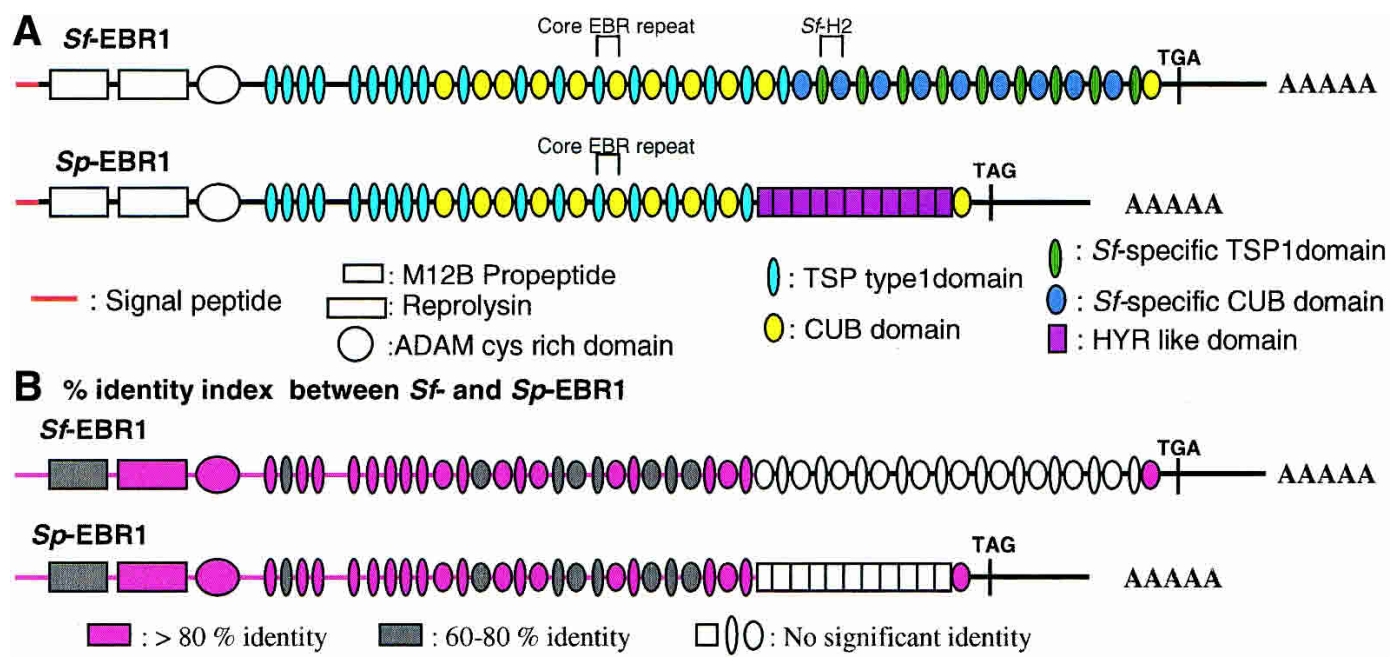

Figure 3. Domain structures and homology index of $S f$-EBR1 and $S p$-EBR1. (A) The horizontal red line shows signal peptides; the vertical line at the right after the last CUB domain shows the stop codon. From the left, the boxes show propeptide, reprolysin, ADAM cis-rich domain, TSP-1 domain repeats, core EBR repeats (alternative TSP-1 and CUB domain repeats; light blue and yellow ovals), and species-specific EBR repeats (dark blue and green ovals; for the $S f$-EBR1) or HYR-like domain repeats (purple boxes; for the $S p$-EBR1). An EBR repeat represents a pair of TSP-1 and CUB (e.g., the set of thin green oval and dark blue oval) of $S f$-EBR1. $(B)$ Graphic representation of the homology between $S f$-EBR1 and Sp-EBR1. Domains shown in pink represent $>80 \%$ amino acid identity; those shown in gray represent $60 \%-80 \%$ identity between $S$. franciscanus and S. purpuratus EBR1 at the amino acid level. No significant identity was observed in the white-colored region. 
ingly similar repeat structure but with some notable differences compared with $S f$-EBR1. The deduced amino acids sequence of $S p$-EBR1 consists of 3713 amino acid residues. Starting at the $\mathrm{N}$ terminus, the protein contains a 30-residue signal peptide followed by a putative propeptide, metalloprotease domain, cis-rich domain, eight TSP-1 domains, and eight and one-half core EBR repeats. Thus, $S p$-EBR1 shares the same domain structure as $S f$-EBR1 for the first $\sim 8 \mathrm{~kb}$ of sequence and shares $88 \%$ nucleotide identity with $S f$-EBR1 in this region (Fig. 3B). This includes the first eight and one-half core EBR repeats, which have $80 \%-90 \%$ identity with the corresponding EBR repeats in $S f$-EBR1. The last CUB domain of both $S f$-EBR 1 and $S p$-EBR1 also shares $86 \%$ identity (Fig. 3B). However, the region of $S p$-EBR1 corresponding to the species-specific Sf-EBR1 domains (dark blue and green ovals or white ovals) contains 11 repeats of a HYR domain (purple boxes or white boxes) and not the homogeneous EBR repeats observed in Sf-EBR1 (Fig. 3A,B). Each HYR repeat is 243 bp or 81 amino acids, and each repeat, except the first and the last HYR repeat, shows $\sim 95 \%$ sequence identity. The HYR repeat domain in $S p$ EBR1 is distinct from the previously reported hyalin gene containing $12 \mathrm{HYR}$ repeats in $S$. pupuratus (Wessel et al. 1998), with $<40 \%$ amino acid identity when compared with HYR-like domains in $S p$-EBR1. We examined the adhesive function of the $S p$-HYR by measuring the adhesion of sperm to recombinant $S p$-HYR expressed as a GST fusion protein. $S$. purpuratus sperm bind specifically to $S p$-HYR compared with $S$. franciscanus sperm and GST controls (Fig. 4). Therefore, the species-unique HYR domain of $S p$-EBR1 functions as a species-specific receptor for sperm adhesion analogous to the speciesspecific EBR repeats of $S f$-EBR1 (Fig. 2C). The deduced EBR1 sequences from both species lack an identifiable transmembrane domain, but EBR1 would be expected to be incorporated into the VE ECM based on its similarity to other ADAMTS proteins (Kuno and Matsushima 1998).

Northern blot analyses were performed with both common and species-specific probes to examine if the probes hybridize to the same size transcript. The common probe derived from the $5^{\prime}$ region of $S f$-EBR 1 detected a single mRNA species of $\sim 14-15 \mathrm{~kb}$ for $S$. franciscanus and 12-13 kb for $S$. purpuratus. Each speciesspecific region of EBR1 hybridized to the same size transcript identified with the $5^{\prime}$ common region probe, but in a species-specific manner (Fig. 5). Reverse tran-

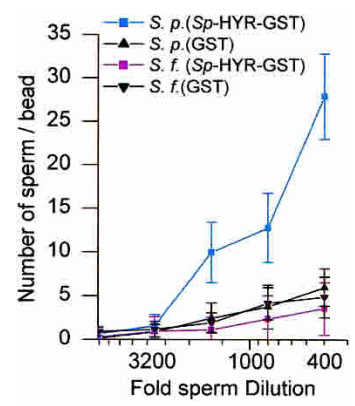

Figure 4. Species-specific binding of acrosome-reacted sperm to immobilized recombinant $S p$-HYR protein. The graph shows the number of sperm binding to the beads. Blue line indicates $S$. purpuratus sperm; red line, $S$. franciscanus sperm. Both black lines show the binding to GST controls.

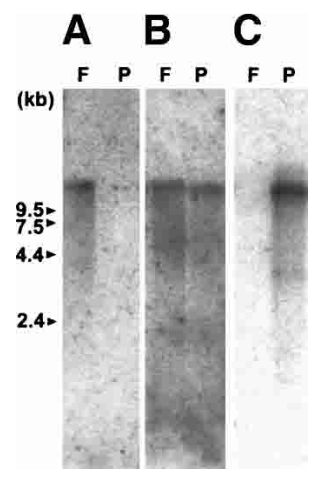

Figure 5. Northern blot analysis of $S$. franciscanus and S. purpuratus ovary mRNAs. (F) S. franciscanus ovary mRNA; (P) S. purpuratus ovary mRNA (1 $\mu \mathrm{g} /$ lane). The probes are as follows: $S f$-EBR repeat $(\mathrm{H} 2)$ DNA $(A), 5^{\prime}$ region (TSP-1 repeats through the first TSPCUB domains) of $S f$-EBR1 $(B)$, and $S p$-HYR-like domain DNA $(C)$.

scriptase PCR (RT-PCR) analysis also showed that the EBR1 gene that was originally cloned from ovary cDNA library exists in unfertilized eggs but is not detected in sperm (data not shown).

We have cloned and sequenced the gene encoding the species-specific egg bindin receptor, EBR1, from both $S$. franciscanus and $S$. purpuratus. These genes are novel ADAMTS-like proteins and share a long repetitive architecture with species-specific domains unique to $S$. franciscanus and S. purpuratus that are situated near the $3^{\prime}$ end. We have also demonstrated that the species-unique EBR1 repeats of $S$. franciscanus and $S$. purpuratus display species-specific adhesive activity by using a variety of different assays. The finding that recombinant EBR1 protein displays species-specific sperm adhesion and bindin-binding activity indicates that protein, but not carbohydrate, is important for determining the species specificity of sperm-egg adhesion. Based on the lack of an identifiable transmembrane domain and the similarity to ADAMTS, it is likely that EBR1 is an ECM protein, which is consistent with the fact that sperm bind to the VE, which represents the ECM of the egg (Glabe and Vacquier 1978; Hirohashi and Lennarz 1998). The repeated structure of the adhesive domain may be important for determining the strength of the adhesive interaction by providing multivalency. This correlates well with the structure of bindin, which has a complementary repeated structure (Minor et al. 1991).

EBR 1 also has a variant ADAMTS domain at its 5' end consisting of a signal peptide, propeptide domain, and zinc metalloprotease domain, but it lacks a disintigrinlike domain. Instead, an ADAM cysteine-rich (ACR) domain, which is typical of ADAM and snake venom proteases, as well as ADAMTS, immediately follows the zinc metalloprotease domain. The zinc metalloprotease domain contains a catalytic site consensus sequence identified in snake venom and matrix metalloproteinases (Bode et al. 1993). This suggests that EBR1 may have protease activity, but further study is required to determine whether this is the case. Although EBR1 lacks a disintrigin-like domain, other ADAMTS family members, such as ADAMTS1 and ADAMTS5, also lack disintigrin domains (Adams and Tucker 2000). Like other ADAMTS members, EBR1 has TSP-1 repeats after the ACR domain. The TSP-1 repeats are found in "matricellular" proteins (Bornstein 2001) and are believed to be 
involved in a variety of cellular processes, including cell and matrix adhesion, cell migration, and sulfated polysaccharide binding. TSP-1 repeats fold into a novel, antiparallel, three-stranded fold capped by disulfide bonds on each end (Tan et al. 2002). After the TSP-1 repeats of EBR1, there are a series of alternating CUB and TSP-1 repeats. Although CUB and TSP-1 domains occur together in other proteins, this is the first instance in which they have been found in a tandemly repeated fashion that we refer to as an EBR repeat. Both the CUB subdomain of the EBR repeat and the HYR domains are predicted to fold as immunoglobulin-like $\beta$-sandwich structures (Bork and Beckmann 1993; Callebaut et al. 2000). The CUB domain protein, spermadhesin, has been implicated in mammalian sperm adhesion by binding to the zona pellucida (Romero et al. 1997). The known adhesive function of both the TSP-1 and CUB domains in other proteins is consistent with their proposed role as specific receptors for bindin in sea urchin fertilization. In $S p$-EBR1, the last 10 species-specific EBR repeats of $S f$ EBR1 are replaced with 11 HYR repeats. Further functional dissection of the individual EBR1 domains may provide some insight into the roles that EBR1 plays in fertilization.

\section{Material and methods}

Animal collection

Sea urchins, $S$. franciscanus and $S$. purpuratus, were obtained from Marinus, Inc.

Generation of Sf-H2 polyclonal antibody

Polyclonal antibodies were produced in female rabbits (New Zealand white) by subcutaneous injection of purified $S f$-H2 GST fusion protein. Anti-Sf-H2 antibodies were purified with protein A Sepharose column chromatography and then applied on a GST column to remove anti-GST antibodies.

\section{Northern blot analysis}

Northern blot analysis was performed with isolated $S$. franciscanus and $S$. purpuratus ovary mRNA probed with ${ }^{32} \mathrm{P}$-labeled $S f-\mathrm{H} 2,5$ 'region (TSP-1 repeats through the first TSP-CUB domains) of $S f$-EBR1, and $S p$ HYR domain DNA (Kamei et al. 2000).

\section{Western blot analysis}

VEs were prepared from both species of sea urchin eggs (Glabe and Vacquier 1977a; Hirohashi and Lennarz 1998) and separated by electrophoresis on $4 \%-20 \%$ SDS-PAGE gradient gels. Each sample was loaded at 2, 5 , and $10 \mu \mathrm{g}$ per lane and detected with 1:2500 dilution of anti-Sf-H2 polyclonal antiserum as the primary and 1:25,000 dilution of HRP conjugated rabbit $\mathrm{F}\left(\mathrm{ab}^{\prime}\right)_{2}$ antibody as the secondary antibody.

\section{Inhibition of fertilization}

The inhibition of fertilization assay was performed with $S$. franciscanus and $S$. purpuratus eggs fertilized with sperm of the same species. After a 10-min preincubation with each concentration of antibody or recombinant protein, samples were inseminated with a concentration of sperm adjusted to give $\sim 70 \%-80 \%$ fertilization in the absence of inhibitor. The fertilization reaction was stopped after $5 \mathrm{~min}$ by the dilution of the sperm with filtered seawater. The fertilized eggs were scored by counting with a stereo microscope (Minor et al. 1993).

Sperm-beads adhesion assay

The sperm-binding assay was performed as previously described (Stears and Lennarz 1997) with glutathione agarose beads loaded with Sf-H2GST fusion protein. GST fusion protein alone was used as a control. The numbers of attached sperm to at least 30 beads were counted by observation with a microscope.
Egg agglutination assay

Egg agglutination assays were performed as previously described (Glabe and Vacquier 1977b; Glabe and Lennarz 1979) by using recombinant Sf-H2 protein as an inhibitor. Agglutination of both species of eggs by homologous bindin was performed with increasing concentrations $(0-40$ $\mu \mathrm{g} / \mathrm{mL}$ ) of recombinant Sf-H2 and a constant concentration of bindin, which gave at least $75 \%$ egg agglutination.

Receptor ligand binding using iodinated EBR1

Recombinant ${ }^{125}$ I-labeled $S f$-H2 protein was iodinated by using Iodogen (Pierce) according to the manufacturer's instructions and was incubated with purified bindin particles for $10 \mathrm{~min}$ in filtered seawater-Tris ( $\mathrm{pH} 7.5)$ at room temperature. The sample was layered on a $20 \%$ sucrose pad in a 0.4-mL Eppendorf tube and centrifuged at $15,000 \times \mathrm{g}$ for $10 \mathrm{~min}$ to separate the iodinated protein bound to bindin particles from the unbound protein. The pellet and supernatant fractions were counted by using a $\gamma$ counter. The nonspecific background was determined by adding an excess of cold recombinant $S f$-H2 protein.

Localization of receptor protein on the egg

For antibody staining, $2.5 \%$ paraformaldehyde $/ 1 \%$ glutaraldehyde-fixed unfertilized and fertilized eggs were used. Fixed eggs were washed with Tris-buffered saline (TBS; pH 7.5) several times, and then blocked with $1 \%$ IgG free BSA in TBS. The samples were incubated with anti-Sf-H2 antiserum at a 1:10 dilution for $1 \mathrm{~h}$, washed three times with $0.1 \%$ Tween-TBS ( $\mathrm{pH} 7.5$ ), and then placed in blocking solution a second time. The TRITC-labeled goat $\mathrm{F}\left(\mathrm{ab}^{\prime}\right)_{2}$ antirabbit $\mathrm{F}\left(\mathrm{ab}^{\prime}\right)_{2}$ antibody was treated at a 1:1000 dilution for $1 \mathrm{~h}$ and washed with $0.1 \%$ Tween-TBS $(\mathrm{pH} 7.5)$ thoroughly, and samples examined under the fluorescence microscope.

cDNA library screening and isolation of the complete cDNA $S$. franciscanus cDNA libraries from sea urchin ovaries were constructed by using $\lambda$ ZAP (Stratagene) with oligo $\mathrm{dT}$ primer or random primer as recommended by the manufacturer. The $S$. purpuratus library was kindly provided by Dr. Wessel (Brown University, Providence, RI). The library was screened by using ${ }^{32} \mathrm{P}$-labeled $S f-\mathrm{H} 2$ or unique $5^{\prime}$ and $3^{\prime}$ DNA fragments as probes. The complete gene sequence of the cDNAs was obtained by cDNA primer walking and the determination of $5^{\prime}$ end of cDNA by the RACE (rapid amplification cDNA ends) analysis for both $S$. franciscanus and $S$. purpuratus. The open reading frame and domain structure was assembled and analyzed by using Lasergene analysis software (DNASTAR Inc; Burland 2000) and SMART search (Letunic et al. 2002).

\section{Acknowledgments}

We thank Dr. V.D. Vacquier for thoughtful discussions and advice, and Dr. G. Wessel for kindly providing the $S$. purpuratus ovary cDNA library. We also thank Dr. J. Claypool for experimental advice and discussions. We thank Young Min Lee and Chae Lee for assistance in screening of the cDNA libraries.

The publication costs of this article were defrayed in part by payment of page charges. This article must therefore be hereby marked "advertisement" in accordance with 18 USC section 1734 solely to indicate this fact.

\section{References}

Adams, J.C. 1997. Thrombospondin-1. Int. J. Biochem. Cell Biol. 29: $861-865$.

Adams, J.C. and Tucker, R.P. 2000. The thrombospondin type 1 repeat (TSR) superfamily: Diverse proteins with related roles in neuronal development. Dev. Dyn. 218: 280-299.

Bode, W., Gomis-Ruth, F.X., and Stockler, W. 1993. Astacins, serralysins, snake venom and matrix metalloproteinases exhibit identical zincbinding environments (HEXXHXXGXXH and Met-turn) and topologies and should be grouped into a common family, the 'metzincins.' FEBS. Lett. 331: 134-140.

Bork, P. and Beckmann, G. 1993. The CUB domain: A widespread module in developmentally regulated proteins. J. Mol. Biol. 231: 539-545.

Bornstein, P. 2001. Thrombospondins as matricellular modulators of cell function. J. Clin. Invest. 107: 929-934. 
Burland, T.G. 2000. DNASTAR's Lasergene sequence analysis software. Methods Mol. Biol. 132: 71-91.

Callebaut, I., Gilges, D., Vigon, I., and Mornon, J.P. 2000. HYR, an extracellular module involved in cellular adhesion and related to the immunoglobulin-like fold. Protein Sci. 9: 1382-1390.

Carroll, E.J.J., Acevedo-Duncan, M., Justice, R.W., and Santiago, L. 1986. Structure, assembly and function of the surface envelope (fertilization envelope) from eggs of the sea urchin, Strongylocentrotus purpuratus. Adv. Exp. Med. Biol. 207: 261-291.

Glabe, C.G. and Lennarz, W.J. 1979. Species-specific sperm adhesion in sea urchins: A quantitative investigation of bindin-mediated egg agglutination. J. Cell. Biol. 83: 595-604.

- 1981. Isolation of a high-molecular-weight glycoconjugate derived from the surface of $S$. purpuratus eggs that is implicated in sperm adhesion. J. Supra. Struct. Cell. Biochem. 15: 387-394.

Glabe, C.G. and Vacquier, V.D. 1977a. Isolation and characterization of the vitelline layer of sea urchin eggs. J. Cell. Biol. 75: 410-421.

. 1977b. Species specific agglutination of eggs by bindin isolated from sea urchin sperm. Nature 267: 836-838.

- 1978. Egg surface glycoprotein receptor for sea urchin sperm bindin. Proc. Nat1. Acad. Sci. 75: 881-885.

Glabe, C.G., Lennarz, W.J., and Vacquier, V.D. 1982. Sperm surface components involved in sea urchin fertilization. Alan R. Liss, New York.

He, Z.Y., Brakebusch, C., Fassler, R., Kreidberg, J.A., Primakoff, P., and Myles, D.G. 2003. None of the integrins known to be present on the mouse egg or to be ADAM receptors are essential for sperm-egg binding and fusion. Dev. Biol. 254: 226-237.

Hirohashi, N. and Lennarz, W.J. 1998. The $350-k D a$ sea urchin egg receptor for sperm is localized in the vitelline layer. Dev. Biol. 204: 305-315.

Hubank, M. and Schatz, D.G. 1994. Identifying differences in mRNA expression by representational difference analysis of cDNA. Nucleic Acids Res. 22: 5640-5648.

Kamei, N., Swanson, W.J., and Glabe, C.G. 2000. A rapidly diverging EGF protein regulates species-specific signal transduction in early sea urchin development. Dev. Biol. 225: 267-276.

Kuno, K. and Matsushima, K. 1998. ADAMTS-1 protein anchors at the extracellular matrix through the thrombospondin type I motifs and its spacing region. J. Biol. Chem. 273: 13912-13917.

Letunic, I., Goodstadt, L., Dickens, N.J., Doerks, T., Schultz, J., Mott, R., Ciccarelli, F., Copley, R.R., Ponting, C.P., and Bork, P. 2002. Recent improvements to the SMART domain-based sequence annotation resource. Nucleic Acids Res. 30: 242-244.

Minor, J.E., Fromson, D.R., Britten, R.J., and Davidson, E.H. 1991. Comparison of the bindin proteins of Strongylocentrotus franciscanus, $S$. purpuratus, and Lytechinus variegatus: Sequences involved in the species specificity of fertilization. Mol. Biol. Evol. 8: 781-795.

Minor, J.E., Britten, R.J., and Davidson, E.H. 1993. Species-specific inhibition of fertilization by a peptide derived from the sperm protein bindin. Mol. Biol. Cell. 4: 375-387.

Ohlendieck, K., Dhume, S.T., Partin, J.S., and Lennarz, W.J. 1993. The sea urchin egg receptor for sperm: Isolation and characterization of the intact, biologically active receptor. J. Cell Biol. 122: 887-895.

Primakoff, P. and Myles, D.G. 2002. Penetration, adhesion, and fusion in mammalian sperm-egg interaction. Science 296: 2183-2185.

Romero, A., Romao, M.J., Varela, P.F., Kolln, I., Dias, J.M., Carvalho, A.L., Sanz, L., Topfer-Petersen, E., and Calvete, J.J. 1997. The crystal structures of two sperm adhesins reveal the CUB domain fold. Nat. Struct. Biol. 4: 783-788.

Ruiz-Bravo, N. and Lennarz, W.J. 1986. Isolation and characterization of proteolytic fragments of the sea urchin sperm receptor that retain species specificity. Dev. Biol. 118: 202-208.

Ruiz-Bravo, N., Earles, D., and Lennarz, W.J. 1986. Identification and partial characterization of sperm receptor associated with the newly formed fertilization envelope from sea urchin eggs. Dev. Biol. 117: 204-208.

Stears, R.L. and Lennarz, W.J. 1997. Mapping sperm binding domains on the sea urchin egg receptor for sperm. Dev. Biol. 187: 200-208.

Summers, R.G. and Hylander, B.L. 1975. Species-specificity of acrosome reaction and primary gamete binding in echinoids. Exp. Cell Res. 96: 63-68.

Swanson, W.J. and Vacquier, V.D. 1997. The abalone egg vitelline enve- lope receptor for sperm lysin is a giant multivalent molecule. Proc. Nat1. Acad. Sci. 94: 6724-6729.

Tan, K., Duquette, M., Liu, J.H., Dong, Y., Zhang, R., Joachimiak, A., Lawler, J., and Wang, J.H. 2002. Crystal structure of the TSP-1 type 1 repeats: A novel layered fold and its biological implication. J. Cell Biol. 159: 373-382.

Tang, B.L. 2001. ADAMTS: A novel family of extracellular matrix proteases. Int. J. Biochem. Cell Biol. 33: 33-44.

Tang, B. and Hong, W. 1999. ADAMTS: A novel family of proteases with an ADAM protease domain and thrombospondin 1 repeats. FEBS Lett. 445: 223-225.

Vacquier, V.D. and Moy, G.W. 1977. Isolation of bindin: The protein responsible for adhesion of sperm to sea urchin eggs. Proc. Natl. Acad. Sci. 74: 2456-2460.

Vacquier, V.D., Tegner, M.J., and Epel, D. 1973. Protease released from sea urchin eggs at fertilization alters the vitelline layer and aids in preventing polyspermy. Exp. Cell Res. 80: 111-119.

Wessel, G.M., Berg, L., Adelson, D.L., Cannon, G., and McClay, D.R. 1998. A molecular analysis of hyalin: A substrate for cell adhesion in the hyaline layer of the sea urchin embryo. Dev. Biol. 193: 115-126.

Yanagimachi, R. 1981. Mechanisms of fertilization in mammals. In Fertilization and embryonic development in vitro (ed. L.M.a.J.D. Biggers), pp. 81-182. Plenum Press, New York.

. 1994. Mammalian fertilization. In The physiology of Reproduction, 2nd ed. (ed. E.K. Neill), pp. 189-317. Raven Press, New York. 


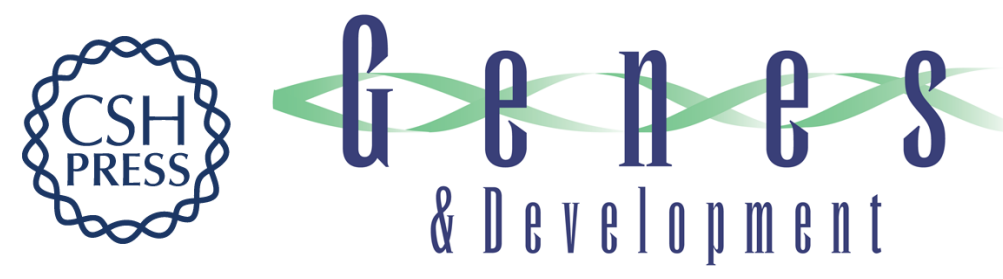

\section{The species-specific egg receptor for sea urchin sperm adhesion is EBR1,a novel ADAMTS protein}

Noriko Kamei and Charles G. Glabe

Genes Dev. 2003, 17:

Access the most recent version at doi:10.1101/gad.1133003

References This article cites 35 articles, 10 of which can be accessed free at: http://genesdev.cshlp.org/content/17/20/2502.full.html\#ref-list-1

License

Email Alerting

Receive free email alerts when new articles cite this article - sign up in the box at the top Service right corner of the article or click here.

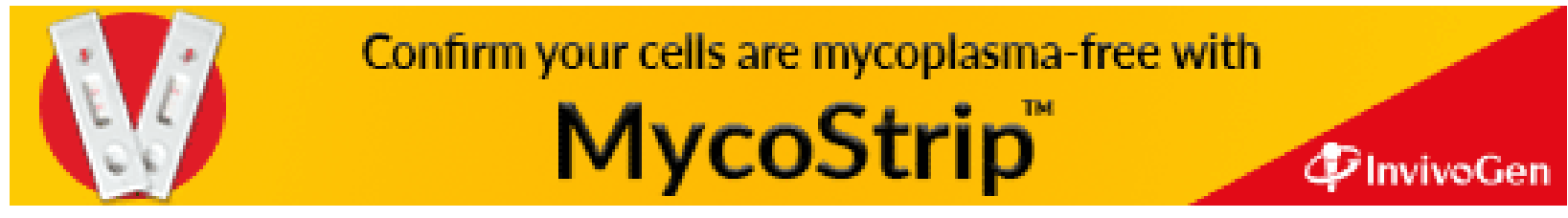

